\section{Globalización, desigualdad y transmisión de las enfermedades tropicales en el Amazonas venezolano}

\author{
Globalization, inequality, and transmission of \\ tropical diseases in the Venezuelan Amazon
}

Carlos Botto-Abella 1,2

Beatriz Graterol-Mendoza 3

\footnotetext{
${ }^{1}$ Facultad de Medicina, Universidad Central de Venezuela, Caracas, Venezuela.

2 Centro Amazónico de Investigación y Control de Enfermedades Tropicales, Ministerio de Salud y Desarrollo Social, Puerto Ayacucho, Venezuela. 3 Estación Experimental Amazonas, Instituto Nacional de Investigaciones Agrícolas, Puerto Ayacucho, Venezuela.

Correspondencia C. Botto-Abella

Instituto de Medicina Tropical, Facultad de Medicina, Universidad Central de Venezuela. Zona Postal 1041-A, Apartado Postal 47623 Caracas, Venezuela. okotoima@yahoo.co.uk
}

\begin{abstract}
Economic globalization appears to be causing greater inequalities and increased vulnerability to tropical diseases around the world. The Venezuelan Amazon population, especially the rural indigenous population, displays among the worst health indicators in the Americas. High infant mortality rates in remote indigenous populations indicate that such communities have been affected by the globalization of disease, rather than favored by globalization of health. Globalization has also influenced public policies in the country, affecting the efficiency of control programs targeting tropical diseases. A new global pact for the sustainable development of the planet is needed, supported by the globalization of human values and rights. In Venezuela, new policies for the indigenous health sector, more resources, and greater autonomy could help reduce the inequities described here in the Venezuelan Amazon.
\end{abstract}

Communicable Diseases; Amazonian Ecosystem; Public Policies

\section{Introducción}

En este trabajo se exploran las relaciones entre el proceso de globalización, las políticas públicas en las Américas y los cambios de orden ambiental y sociocultural ocurridos en las últimas décadas en el ámbito de comunidades locales indígenas en el Estado Amazonas de Venezuela, así como su impacto sobre la salud pública.

Se empleará el término globalización en el sentido utilizado por CEPAL (Comisión Económica para América Latina y el Caribe) ${ }^{1}$ (p. 13), haciendo referencia a la "creciente gravitación de los procesos financieros, económicos, ambientales, políticos, sociales y culturales de alcance mundial en los de carácter regional, nacional y local", y especialmente a su dinámica determinada en gran medida por el carácter desigual de los actores en juego.

La dimensión económica y financiera de este proceso está vinculada al desarrollo de las empresas transnacionales, con la liberalización de las transacciones económicas internacionales de capitales ${ }^{2}$. Para Feo-Isturiz ${ }^{3}$ el rasgo fundamental de la globalización es la imposición de una forma de pensamiento y una forma de concebir el mundo, la sociedad, la producción y las relaciones entre las naciones, que se conoce como neoliberalismo. Esta globalización del mercado crea a veces oportunidades originales para algunos países y muchas más entran en conflicto y tensión con la "globalización de los valores", sus- 
tentada en la Declaración Universal de los Derechos Humanos y en la Declaración del Milenio de las Naciones Unidas ${ }^{4}$. Asimismo, la revolución de las comunicaciones y la globalización de la información plantean posibilidades inéditas de desarrollo inconcebibles pocos años atrás, pero a la vez amenaza la preservación de la identidad cultural en los niveles locales. Por otra parte, el impacto de todos estos procesos en el ambiente y en el clima del planeta es de enorme relevancia en la emergencia de nuevas enfermedades y en el resurgimiento de antiguas endemias 5,6.

El presente trabajo pretende describir las fuertes asimetrías que existen en la distribución de los recursos y perfiles de salud entre diferentes localidades de una misma región y arrojar luz sobre algunas relaciones de los procesos de cambio global con la transmisión de enfermedades en comunidades amerindias.

Estas relaciones pretenden ser analizadas desde una perspectiva que considera esencial la historia de la región y sus cambios.

\section{El choque de dos civilizaciones y la salud pública en la época colonial y postcolonial}

La globalización no es un fenómeno nuevo: hace cinco siglos vivió un episodio muy importante cuando se produjo el choque de civilizaciones de Europa y América 7 . En Tenochtitlán en el año de 1520, durante el asedio de la ciudad por los españoles, se estima que murieron 1,75 millones de indígenas a consecuencia de la introducción de la viruela y otras enfermedades, según relatos de los Informantes Anónimos de Tlatelolco ${ }^{8}$. La población indígena de México, estimada en 18 millones de habitantes antes de la llegada de los españoles, se redujo a un $2,7 \%$ de la población original y la población indígena de Brasil, estimada en 4 millones en el año 1500, se había reducido $99 \%$ en 19709 .

Para hallar un punto de comparación en cuanto a letalidad en el continente europeo hay que remontarse a la Edad Media, cuando la peste bubónica, importada del Asia Central en 1347, produjo 25 millones de muertes, con una reducción de la población europea de un $66 \%$. Enfermedades como la tuberculosis, la sífilis, la viruela, el sarampión, el cólera y la peste azotaban en forma endémica o epidémica a una buena parte de la población europea hacia el año 1700, determinando que la expectativa de vida fuera muy corta 9,10 .

La mayor parte de estas enfermedades fueron importadas durante la conquista y la colonia desde el continente europeo, pero a ellas debe agregarse una larga lista, entre las que destacan la malaria, la fiebre amarilla y la oncocercosis, que fueron importadas probablemente desde la costa occidental de África a través del tráfico de esclavos 11,12,13,14.

En el siglo XIX la viruela produjo numerosas muertes en Caracas. La Gazeta de Caracas (Diciembre, 1817) 15 (p. 76) describe el impacto que produce la enfermedad: "Cuando aparece la viruela, todos los que no están libres de sus estragos, huyen del lugar infecto; cesan las comunicaciones; el comercio desaparece; la agricultura de sus inmediaciones se ve abandonada, y los males que se siguen a estas causas son incalculables en sus resultados". Hacia el año 1830 "enfermedad y hambre frenan un mayor crecimiento poblacional interno venezolano" 15 (p. 981). La malaria domina en los llanos, la fiebre amarilla es endémica y se extiende en forma epidémica en 1856 por todo el país afectando a las principales ciudades. En 1854 aparece además una epidemia de cólera, y se paraliza la vida pública y política. "Ni las guerras que entonces asolan a nuestros pueblos dañan tanto a la población y a la economía nacional como esta enfermedad que en la pueblerina Caracas deja más de dos mil muertos" 15 (p. 981).

A todos los males mencionados se sumaron en este período los producidos por las guerras, no sólo la lucha emancipadora, sino también diversas guerras civiles de las cuales solamente una, la Guerra Federal (1858-1863) produjo más de cien mil muertos, además de "aumento directo de la tasa de mortalidad como secuela de hambrunas y desarraigos por las perturbaciones sociales y las crisis económicas" 15 (p. 982).

Para esa época el impacto combinado de los "gérmenes" importados de Europa y de África y los enfrentamientos armados había diezmado o aniquilado completamente muchas poblaciones amerindias venezolanas. A fines del siglo XIX los indígenas "reducidos a poblados" tienen una población decreciente, y se enfrentan a la pérdida de sus tierras a manos de los colonos y pobladores criollos. Aunque un Decreto de Simón Bolívar en 1820 ordenó el reintegro de tierras a las comunidades indígenas reducidas en "resguardos", este no se cumplió, y hacia 1828 los indígenas reducidos "se han dispersado en gran parte, abandonando las poblaciones y sumiéndose de nuevo en los bosques" 15 (p. 133-4).

\section{Las políticas y la salud pública a comienzos del siglo $\mathrm{XX}$}

Las políticas públicas hacia el Sector Salud en las Américas a comienzos del siglo XX fueron fuertemente influidas por las investigaciones y cam- 
pañas de control de la anquilostomiasis y de la malaria, iniciadas en Brasil en el año 1915, con el apoyo de la Fundación Rockefeller 16, en México en 192717 y en otros países de la región. El descubrimiento de la naturaleza vectorial de la malaria, el hallazgo de una "bala mágica" (el DDT) para interceptar al vector infectado y el convencimiento del poder de la tecnología occidental para transformar al mundo, llevó a la Organización Mundial de la Salud (OMS) al diseño de la más ambiciosa estrategia global para erradicar una enfermedad jamás concebida 18 . La campaña mundial emprendida contra la malaria en la posguerra tuvo características inusuales: utilizó "armas químicas” probadas en la segunda guerra mundial y una organización vertical "militar". Venezuela se incorporó tempranamente a esta "batalla" bajo el liderazgo de Arnoldo Gabaldón, quien desde 1936 se hallaba al frente de los programas de control en Venezuela y formó parte del Comité de Expertos de la OMS desde su creación, lo que le ayudó a lograr la erradicación de la malaria en la más grande área tropical reportada hasta esa fecha ${ }^{19}$. No obstante, la creación de Malariología y Saneamiento Ambiental como una estructura organizativa relativamente independiente dentro del Ministerio de Salud y los conflictos entre la verticalidad de los programas de erradicación de la malaria y la horizontalidad requerida por la atención primaria de Salud impulsada por la OMS, tuvieron importantes repercusiones en el día a día de los servicios de salud.

Sin dejar de enaltecer la profunda pasión humanista que guió a los artífices de estas luchas en cada uno de los países, Packard 18 analiza agudamente el conflicto entre los intereses nacionales y las políticas imperantes en la región de las Américas en plena época de la guerra fría. Birn 17 describe como México fue utilizado como un laboratorio de investigación científica por la Fundación Rockefeller, aunque ésta no se involucró en la campaña de control de la malaria en ese país. Así, puede afirmarse 18 que el control de las enfermedades tropicales fue también un medio para expandir mercados, vender productos manufacturados (entre ellos los insecticidas) y asegurar materia prima para la potencia dominante en la región.

\section{Las políticas públicas en las Américas en los años 90}

Sin lugar a dudas, las políticas mundiales en el decenio de los años 90 han modelado de manera importante las políticas nacionales hacia el sector salud. En esta región, los programas de ajuste macroeconómico propugnados por los organis- mos internacionales fueron orientados hacia la apertura de las economías a la competencia externa, para dar mayor participación al capital privado ${ }^{20}$. No obstante, existe creciente preocupación sobre el hecho que estos cambios, basados en la eficiencia de los servicios, que redujeron el papel del Estado para proveer servicios de salud, han conducido en muchos países pobres a una mayor inequidad e injusticia social 21. Después de una década se ha observado en muchos países de la región deterioro del ingreso, y empeoramiento de la concentración de la renta, "afectando la cohesión social y la gobernabilidad, con un concomitante aumento de las desigualdades y de la violencia" 20 (p. 909).

La "Nueva Agenda” apoyada por el Banco Mundial proponía una mejor utilización de los escasos recursos, que deberían ser "costo-efectivos" y estar dirigidos a aliviar "la carga de la enfermedad" ("burden of disease"). De esta forma, los servicios debían ser redireccionados a los que no pueden satisfacer sus necesidades en el mercado, privilegiándose los servicios básicos y de bajo costo, con mayor cobertura y eficacia 20 .

La descentralización funcional era un componente importante de la reforma, con la cual se esperaba "lograr mayor transparencia y equilibrios financieros, mejorar la eficiencia en el uso de los recursos (...) y ampliar la cobertura de dichos sistemas" 20 (p. 922). Sin embargo, su implementación entrañó severos conflictos con los principios de solidaridad y equidad que habían guiado en la región de manera tradicional la organización de los sistemas de servicios 20.

La desigualdad en el mundo exhibe hoy "niveles absurdos y tendencias ambiguas” 2 (p. 31). Los indicadores de desarrollo humano que resumen un conjunto de índices basados en la esperanza de vida, la salud pública, el nivel de educación y los ingresos por habitante, muestran las enormes diferencias que existen entre países pobres y ricos, y también que en la década de los 90 , un grupo de más de veinte países experimentaron un retroceso en estos indicadores 3 , lo cual indica un fuerte desencuentro entre la salud y el modelo económico. El planeta tierra muestra en conjunto un Índice Gini que revela mayor desigualdad que en el país más desigual. Veinticinco millones de los americanos más ricos tienen más ingresos que los dos mil millones más pobres de la tierra, y las desigualdades en la mortalidad infantil también se han incrementado abruptamente: la probabilidad de morir para un niño menor de cinco años en el África subsahariana, que era 19 veces más alta que en un país rico en el año 90, ahora es 26 veces mayor 4 .

En América Latina y el Caribe el número de personas que viven con menos de un dólar dia- 
rio aumentó de 48 a 57 millones en la década de los 90 4, y entre 1980 y fines de los 90 el número total de pobres pasó de 135 a 211 millones. Como señala Brundtland 22 , el contraste entre los "have" y "have not" es asombroso. Ciento setenta millones de niños en el mundo están desnutridos y tres millones mueren cada año como resultado, mientras que un billón de adultos tienen sobrepeso, 300 millones son clínicamente obesos y medio millón morirán por enfermedades asociadas. "Unos no tienen elección, y otros hacen la elección errónea” 22 (p. 9). La globalización ha hecho también que se conozcan mejor estos hechos, y que la conciencia de la desigualdad aumente.

Desde la Conferencia de Alma Ata (1978) ( $S a-$ lud para Todos en el Año 2000) hasta la Declaración del Milenio de las Naciones Unidas (2000) se ha avanzado hacia el establecimiento de: (a) un conjunto de metas mundiales relacionadas con la salud pública, la seguridad alimentaria y la educación, suscritos por todos los países, y al mismo tiempo; (b) un compromiso de todas las naciones para modificar las reglas del juego, a través de la aplicación de nuevos enfoques para el alivio de la deuda, mediante la eliminación de subsidios y medidas discriminatorias que permitan expandir el comercio sobre una base de mayor equidad y a través de la transferencia de tecnologías 4.

Sin embargo, a pesar de los compromisos y declaraciones, y aunque existe mayor conciencia en el mundo de la desigualdad y mejores condiciones para luchar contra ella, esta ha aumentado de manera notable.

\section{Las políticas públicas en el Amazonas venezolano}

En los últimos cincuenta años han ocurrido en el Amazonas venezolano, como consecuencia del proceso de globalización, cambios vertiginosos en comunidades indígenas locales que tenían poco o ningún contacto con el estado venezolano y la sociedad occidental pocas decenas de años atrás.

Las políticas públicas se expresaron en un conjunto de programas que impulsó el Estado venezolano y que impactaron dramáticamente la vida de los pueblos indígenas 23 , algunos de los cuales iniciaban en los años 50 un contacto más permanente con el resto de Venezuela.

En forma esquemática pueden reconocerse cuatro fases 24 en la historia reciente de estas relaciones.

\section{La Conquista del Sur}

Las relaciones más intensas entre el Estado Venezolano y la población indígena de Amazonas se iniciaron en 1969 con la creación de la Comisión para el Desarrollo del Sur (CODESUR), que tenía un enfoque económico desarrollista sin ninguna consideración de sustentabilidad, y un enfoque intregracionista en relación con la población indígena 24,25 .

A nivel del Amazonas la oferta de bienes y servicios del Estado y la construcción de viviendas contribuyó a sedentarizar a la población indígena, desvinculándola de sus sistemas tradicionales de explotación y manejo de los recursos naturales 26. Aquella era visualizada a menudo como "un problema", para el cual se ofrecían "soluciones", como la integración nacional a través de la salud, la educación, la construcción de viviendas, y vialidad y la asignación de tierras. El discurso subyacente suponía maximizar el beneficio de manera que los escasos recursos llegaran a la mayor cantidad de gente posible. Sin embargo, los cambios inducidos por estas políticas y por la presencia de misiones religiosas, provocaron una modificación del patrón de asentamiento, con aumento de la densidad poblacional por migraciones internas, asociado con agotamiento de los recursos naturales e incremento de la desnutrición.

Hasta 1960, el Estado Venezolano desarrolló a nivel nacional políticas de salud con un enfoque preventivo, e iniciaron actividades distintas Programas verticales de control de enfermedades tropicales. El ejemplo paradigmático está representado por el Programa de Control de la Malaria, que había logrado importantes éxitos en el país, gracias a una visión sanitarista y preventiva, y a la existencia de importantes ingresos petroleros que sustentaban la campaña de erradicación de la malaria 27. En años posteriores (1960 en adelante) el modelo sanitarista preventivo se "movió" hacia un modelo hospitalario y curativo, basado en las expectativas de la población y en la posibilidad de satisfacerlas a través de los altos ingresos petroleros, en lo que se denominó "fase curativa de altos ingresos" 27 (p. 18).

\section{Políticas conservacionistas}

Con la creación de la Organización de Países Exportadores de Petróleo (OPEP) y el incremento de los precios del petróleo en un 400\%, los ingresos del país casi se cuadruplicaron y la explotación de recursos naturales en el sur de Venezuela dejó de ser una prioridad. A fines de los años 70, con el cambio de gobierno, y bajo la influencia de la Conferencia de las Naciones Unidas de Estocol- 
mo (1972) se creó el Ministerio del Ambiente y los Recursos Naturales Renovables, y este absorbió muchas de las funciones de CODESUR, que desapareció 24,25. En este período se aprobaron numerosas leyes y decretos orientados a la protección ambiental y al desarrollo sustentable y se crearon numerosas áreas bajo régimen de administración especial. En 1978 se aprobó el Decreto 2.552 que prohibió la explotación comercial del bosque tropical en el Estado Amazonas y en 1989 el Decreto 260 que prohíbe la explotación minera en la entidad territorial 26 . Se crearon además 4 Parques Nacionales (5.953.740ha), 4 Monumentos Naturales y una Reserva de Biosfera de 8.400.000ha 28 .

A partir de 1980 con la disminución del precio del petróleo y la devaluación del bolívar, se inician de nuevo las presiones para la explotación de los recursos naturales en el Amazonas. En el sector salud, a pesar de la influencia de la Conferencia de Alma Ata (1978) y de la promoción por la OMS de la Atención Primaria en Salud, comienza en estos años el dominio de un modelo curativo hospitalario "de reducidos ingresos petroleros" 27 (p. 18).

\section{EI "Gran viraje"}

Entre 1989 y 1992, bajo la segunda presidencia de Carlos Andrés Pérez se aplica en el país un plan de ajuste estructural 29 impulsado por el Fondo Monetario Internacional y el Banco Mundial, el cual contemplaba la Reforma del Estado con la descentralización administrativa como uno de sus componentes ${ }^{30}$. La Reforma era parte de un plan de apertura económica y globalización del mercado, que identificó en Amazonas el progreso con la urbanización y la minería. La descentralización fue visualizada, en esta región, como la redistribución de los ingresos petroleros no asociada a nuevas responsabilidades. En el nivel central el tamaño del estado no disminuyó lo esperado, pero en muchos campos se perdieron rectorías esenciales, y no se crearon en las regiones las capacidades para asumir los nuevos retos que entrañó el cambio descrito. La transformación del Territorio Federal Amazonas a Estado (1992) y la creación de nuevos Municipios, en algunos casos, sin población que justificara su existencia, incrementó de manera notable la burocracia gubernamental y el gasto público en la región, con diversas distorsiones de la economía local. Aunque el gasto público en Amazonas aumentó a más del doble en ese período, más del $80 \%$ era consumido en burocracia y políticas clientelistas 24 , con merma y deterioro de muchos servicios prestados 30 . No obstante, la elección de gobernadores y alcaldes significó un cambio irreversible en la cultura política de los estados y la aparición de nuevas tensiones entre los gobiernos locales y el gobierno central ${ }^{31}$. En el ámbito nacional, el Plan fracasó ostensiblemente en términos de reestructuración económica, y se asoció a una disminución del ingreso per cápita, a un incremento de la pobreza y a turbulencias políticas con diversos intentos de golpes de estado. Desde el punto de vista de la salud corresponde a lo que Briceño-León 29 (p. 18) denomina "fase curativa de bajos ingresos", donde domina la atención hospitalaria curativa, que consumió mas del $70 \%$ de todos los recursos del Ministerio de Sanidad y Asistencia Social.

\section{Política de reconocimiento de los Derechos} de los pueblos Indígenas

Se extiende desde 1999 hasta el presente, enmarcado dentro del proceso de transformación política que protagoniza Venezuela desde la elección de Hugo Chávez como presidente. Este período se extiende desde 1999 hasta la época actual, y comprende dos fases claramente diferenciadas: (a) desde la elección hasta el Golpe de Estado y paro petrolero (1999-2002), y (b) después de estos acontecimientos (2003 al presente).

La primera fase (1999-2002) se caracterizó por la aprobación por referéndum de una nueva constitución (1999) que reconoció los derechos de los pueblos indígenas en un capítulo especial, de la Nueva Constitución (1999) y por una notable participación de líderes y organizaciones indígenas en los asuntos de estado, en un terreno que les estaba casi completamente vedado. Arvelo-Jimenéz 32 destaca la participación de seis constituyentes indígenas en la redacción de la nueva Constitución. Desde el punto de vista de la salud, esta es reconocida como un derecho social fundamental que el Estado venezolano debe hacer cumplir con participación social en el control de la gestión, y se intenta implementar un modelo de atención integral en salud, con una dimensión preventiva importante. De acuerdo con la nueva Constitución, se trata de construir un sistema integrado, descentralizado y financiado con recursos públicos.

Durante la segunda fase (desde 2003) hay una reorientación de la política social con modificaciones parciales de la estrategia anti-pobreza. La inclusión social se transforma en una estrategia central, a través de la cual se esperan reducir progresivamente las inequidades y generar articulación entre política social y económica, y procesos de descentralización y participación. Se crean Planes y Programas especiales, denominados Misiones para la seguridad alimentaria y 
la inclusión social (en el área económica, social, educativa y de salud) 33 .

Las misiones educativas tienen como objetivo enfrentar la exclusión educativa, por ello se desarrolló desde 2003 un Plan masivo de alfabetización y posterior culminación de la escuela primaria y secundaria. En Octubre del año 2005, Venezuela fue declarada por la Organización de las Naciones Unidas para la Educación, la Ciencia y la Cultura (UNESCO) Territorio Libre de Analfabetismo, al sobrepasarse la meta de un millón de personas alfabetizadas en un año (Misión Robinson; http://www.misionrobinson.gov.ve/ accedido el 20/Sep/2006).

El Programa de Atención Integral en Salud incorporó médicos en labores preventivas, de diagnóstico y trabajo social, a nivel de barrios y comunidades con el Plan Barrio Adentro I, que beneficia a millones de personas que estaban excluidas del sistema de salud ${ }^{33}$.

Estos cambios son en muchos casos contrarios a los impulsados por la globalización y la agenda neoliberal, y tiene puntos de contacto con la reforma del sistema de salud en Brasil, que propuso consolidar un "sistema de salud público, universal y descentralizado, basado en la concepción de salud como derecho de ciudadanía y deber del Estado" 20 (p. 22).

Persiste, sin embargo, una enorme brecha entre los derechos establecidos en la Constitución y en las leyes, que reconocen la salud como un derecho ciudadano, y los indicadores de salud que muestran al Amazonas como una de las más postergadas del país.

\section{La tendencia del desarrollo humano en el Amazonas venezolano}

Cuando se analizan las tendencias del desarrollo humano en un período de 25 años en América Latina, puede observarse que Venezuela presentó el menor crecimiento $(0,054)$ entre 19 países de la región, y fue ampliamente superada por países que 25 años atrás mostraban menor Índice de Desarrollo Humano (IDH) que Venezuela 2.

El proceso descrito más arriba se expresa en un empobrecimiento mantenido de Venezuela por lo menos desde 1978, con deterioro persistente de los ingresos e incremento de la deserción escolar, que es más sistemático e intenso en el interior del país comparado con la región capital 34 .

Debe destacarse que este IDH desplaza a la producción per cápita como sinónimo de desarrollo de los países 2 (p. 41), “conjugando una vida larga, logro escolar e ingresos”. El índice combina dos indicadores estructurales (esperanza de vida y analfabetismo) que son el resultado de políticas públicas desarrolladas desde décadas atrás, y que han presentado un aumento sostenido en los últimos 25 años, ubicando a Venezuela para el año 2000 por encima del promedio de América Latina. Por otro lado, el índice incluye dos indicadores coyunturales (tasa de matriculación en la enseñanza e ingresos) que ubican a Venezuela por debajo del promedio del continente para este período 2 .

Para el año 1990 Venezuela presentaba un IDH alto $(0,86)$, pero sufre un deterioro progresivo hasta los años 1996-1998, que lo ubican en un IDH medio $(0,69)$ (Figura 1). En los años siguientes se observa un incremento progresivo para alcanzar nuevamente en el año 2005 un IDH alto $(0,81)$ (Figura 2). Como puede observarse, el Producto Interno Bruto de Venezuela creció (expresado en miles de millones de US\$) desde 1997 hasta el año 2002, cuando se registra una abrupta caída después del paro petrolero, y una recuperación en el año 2004. Estas variaciones en los indicadores macroeconómicas son seguidas en términos generales por las modificaciones en el IDH, lo cual refleja el gasto social realizado principalmente en educación y salud.

El análisis diferencial de este conjunto de índices por entidad federal revela que no solamente se observó una tendencia general al descenso entre los años 1991 y 2000, sino que las brechas entre los estados con mayor y menor potencial de desarrollo se profundizaron, destacándose que los estados con mayor deterioro fueron Delta Amacuro, Amazonas, y Apure, todos ellos con importante población indígena. El IDH de Amazonas mostró en ese período notables diferencias con los valores promedio del país que tienden a acentuarse al final de ese lapso (Figura 1), lo cual indica que había en esta región para el año 2000 mayor exclusión social y menos personas con acceso a oportunidades básicas de educación, salud y trabajo 2 .

Esta situación esta cambiando lentamente, no sólo a través de la inversión en salud y educación, sino también en el área de seguridad alimentaria, microeconomía, desarrollo de cooperativas e inversión en el área cultural, comunicaciones y ciencia y tecnología.

\section{El perfil epidemiológico del Amazonas venezolano}

Como resultado el Amazonas venezolano, cuya población indígena representa más de la mitad de la población del estado, es "otro país" como afirman sus habitantes, donde los niveles de saneamiento ambiental y el porcentaje de la pobla- 


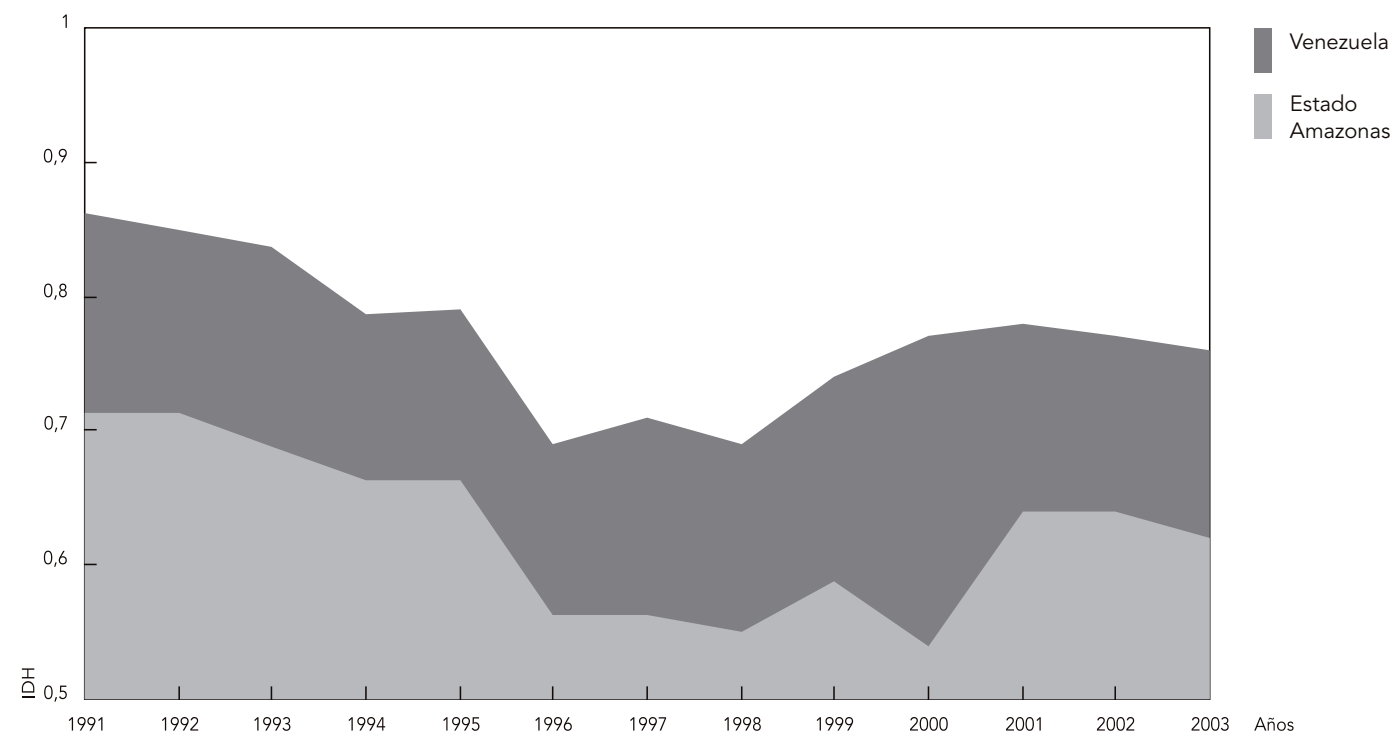

Fuente: Indice de Desarrollo Humano, Venezuela, 2002; Instituto Nacional de Estadística, República Bolivariana de Venezuela (2001-2003).

ción con acceso a agua potable, son significativamente más bajos que en el resto de Venezuela 35 . Como consecuencia, la mortalidad infantil por mil nacidos vivos que alcanzó 19.7 por mil en el año 2000 en el país sube a 38.4 por mil en el Amazonas y la mortalidad materna fue más del doble más alta en Amazonas para ese mismo año 36.

Aunque la mortalidad infantil en Amazonas ha descendido de manera notable en el año 2005 de 38,4 a 31,6 por mil 37 persiste una enorme brecha.

La diferencia en la tasa de mortalidad infantil con el resto del país es producida principalmente por problemas nutricionales y enfermedades transmisibles, que se encuentran entre las principales causas de morbilidad y mortalidad en la población indígena. Como puede apreciarse en la Tabla 1 la mortalidad por enfermedades infecciosas es tres veces y media más alta en Amazonas.

El IDH de Amazonas lo ubica por debajo de varios países africanos con serios problemas de salud. Las tasas de enfermedad diarreica, neumonía y tuberculosis fueron también más altas en el Estado Amazonas, comparado con el resto del país en el año 2000 35,36 y la incidencia de malaria en el año 2003 fue setenta veces más alta 38.
Para este año las cinco primeras causas de morbilidad en el Estado Amazonas están representadas por patología infecciosa: malaria, diarrea, síndrome viral, helmintiasis y rinofaringitis aguda 39 .

Las causas de mortalidad general muestran en cambio una combinación de enfermedades carenciales e infecciosas (desnutrición, diarrea, neumonía, tuberculosis, malaria, VIH-SIDA) y de enfermedades asociadas generalmente al mundo desarrollado (enfermedad cardiovascular, cáncer, accidentes, diabetes, suicidio). Esta "doble carga" propia de los estilos de vida de la abundancia y de la escasez, se observa cada vez con mayor frecuencia en los países del mundo en desarrollo 22 y en poblaciones indígenas 40 .

Si bien las cifras presentadas muestran un enorme contraste con la "Venezuela desarrollada”, estos contrastes se agudizan cuando se examinan las diferencias entre diversas regiones del Estado Amazonas. Destaca la región del Alto Orinoco, donde habita la población yanomami, que en más de un $70 \%$ muestran un patrón de asentamiento muy disperso, y está fuera de la cobertura del sistema de salud, exceptuando las visitas esporádicas de programas de control como 
Figura 2

Índice de Desarrollo Humano (IDH) y Producto Interno Bruto (PIB) en Venezuela.

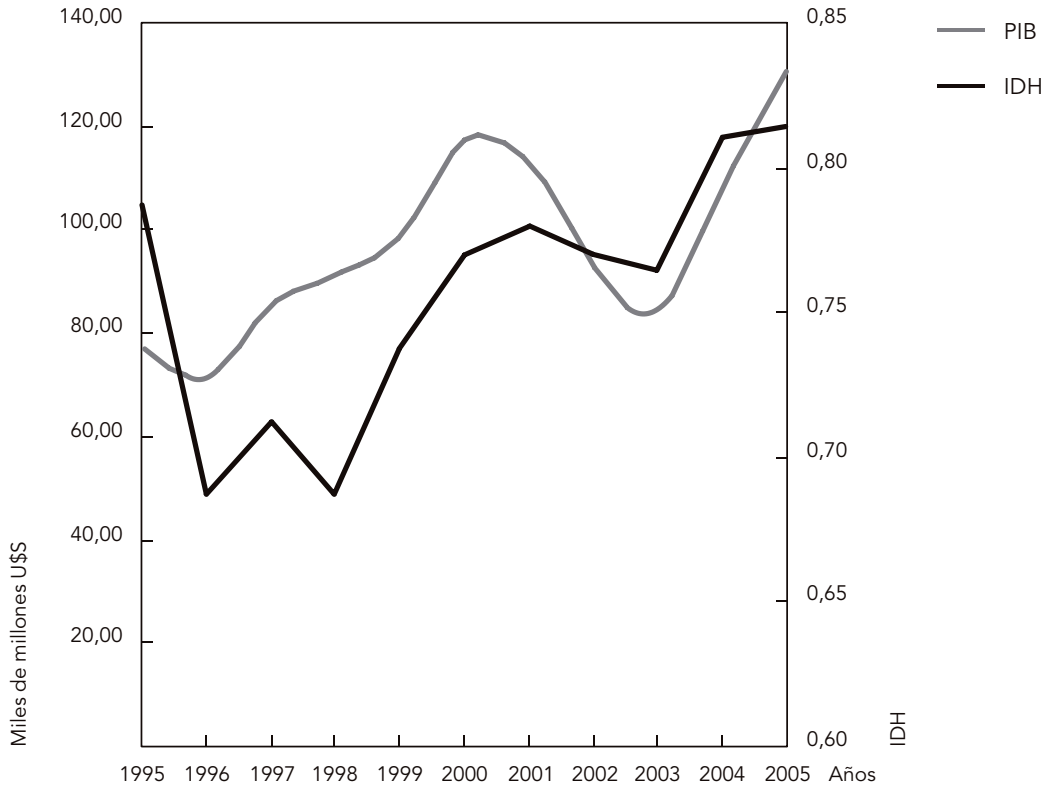

Fuente: Programa de las Naciones Unidas para el Desarrollo 2; Instituto Nacional de Estadística, República Bolivariana de Venezuela (1998-2005); Banco Interamericano de Desarrollo, 2006.

Tabla 1

Desarrollo humano e indicadores de salud en Venezuela y el Estado Amazonas.

\begin{tabular}{lcccccc}
\hline País/Estado & IDH & $\begin{array}{c}\text { Saneamiento } \\
\text { ambiental * } \\
(\%)\end{array}$ & $\begin{array}{c}\text { Agua } \\
\text { potable * }\end{array}$ & $\begin{array}{c}\text { Mortalidad } \\
\text { infantil **, } \star \star \star\end{array}$ & $\begin{array}{c}\text { Mortalidad } \\
\text { materna **,\# }\end{array}$ & $\begin{array}{c}\text { Mortalidad } \\
\text { enfermedades } \\
\text { infecciosas }\end{array}$ \\
\hline Venezuela & 0,75 & 68,0 & 83,0 & 19,7 & 60,1 & 24,7 \\
Amazonas & 0,58 & 21,6 & 40,2 & 38,4 & 165,6 & 88,7 \\
\hline
\end{tabular}

* Informe sobre Desarrollo Humano Global, Venezuela (2000); Programa de las Naciones Unidas para el Desarrollo 35.

** Ministerio de Salud y Desarrollo Social 36.

*** Tasa por 1.000 nacidos vivos;

\# Tasa por 100 mil nacidos vivos.

el de la oncocercosis y el programa ampliado de inmunizaciones.

Cuando se examinan la mortalidad infantil en menores de un año, puede observarse en el año 2000 que las cifras pasan de 19,7 por mil en el nivel nacional a 38,4 por mil en el nivel de estados y a 166,7 por mil a nivel del Alto Orinoco 41. Las cifras de mortalidad en menores de un año en el Alto Orinoco han oscilado entre 250 por mil y 76,9 por mil con tendencia a la disminución entre los años 1998 y 2001 41. En algunas áreas, este podría ser el efecto de mejoras en la atención primaria en salud asociadas a la ejecución de un programa de control basado en el diagnóstico y tratamiento precoz de la malaria y más recientemente por el uso de mosquiteros impregnados 
con piretroides. Como resultado, los índices esplénicos y niveles de hemoglobina mejoraron de manera significativa 42 .

Sin embargo, como anota Kelly 41 la tasa de mortalidad para menores de un año del Alto Orinoco es 4 a 12 veces más alta que la tasa nacional, y 2 a 7 veces mayor que la tasa de Amazonas. Asimismo, entre las primeras causas de mortalidad general en el Alto Orinoco en diversos períodos 11,41 se encuentran la malaria, la diarrea, la infección respiratoria aguda y la hepatitis, enfermedades todas ellas prevenibles y/o tratables, lo cual revela la magnitud de la falta de cobertura del sistema de salud.

De esta forma, las inequidades y las marcadas diferencias en las oportunidades de acceso a bienes y servicios observadas dentro de Venezuela, se repiten en el interior del Estado Amazonas.

\section{Los programas de control de enferme- dades endémicas en Amazonas}

Desde el comienzo de la campaña de erradicación de la malaria, Amazonas fue definida como un área con focos de "malaria inabordable", difíciles de controlar, debido al comportamiento exofílico del vector (Anopheles darlingi) y al carácter seminomádico de la población humana. A pesar de la instauración del diagnóstico y tratamiento temprano, de la fumigación de viviendas y más recientemente del uso de mosquiteros impregnados con piretroides, en los últimos diez años el promedio se estableció en 4666,7 casos por año. A pesar de ello, en el año 2003 la tasa de incidencia malárica se duplicó, alcanzando la cifra de 87,7 casos por mil, más de setenta veces por encima de la tasa nacional (1,2 por mil) 38 . Esto la convirtió en la primera causa de morbilidad en el Estado y en la séptima causa de muerte en los niños menores de un año.

Aunque la resistencia de los agentes de la malaria a la cloroquina y otros fármacos está presente en Amazonas desde muchos años atrás 42 , y la influencia del Fenómeno del Niño ha sido bien demostrada en Venezuela ${ }^{43}$, este cambio notable en la dinámica de transmisión parece haber sido menos influido por factores de orden biológico que por factores de orden social, entre los que destacan:

- Una descentralización incompleta en el sector salud, la cual debilitó el fuerte programa centralizado, vertical, de nivel nacional encargado del control de la malaria, con el fin de incorporar las actividades de control dentro de programas horizontales de Atención Integral de Salud. Al mismo tiempo, el proceso de descentralización iniciado en Amazonas se revirtió, y la aplicación reciente de un sistema centralizado de distribución de los recursos, con pérdida de la autonomía financiera de los organismos de control, contribuyó a la pérdida de la capacidad operativa en el nivel regional.

Rodríguez-Ochoa 44 (p. 421) señalaba como un serio problema del sistema de salud la "grave centralización de todos los niveles decisorios en la capital y en la Dirección regional de Salud". Toro-Landaeta 45 señaló la orientación hospitalario-curativa del modelo y la centralización de los recursos humanos y decisiones en Puerto Ayacucho. Armada 46 (p. 301) resaltó "el peso del carácter centralista imperante en la estructura política y económica del país". A pesar de la implantación de un nuevo modelo de Atención Integral en Salud, los problemas aquí señalados años atrás continúan gravitando de manera decisiva en la persistencia de la malaria y otras enfermedades endémicas en Amazonas.

- Malaria influida por la minería de oro ilegal. Otro factor de importancia lo constituye el resurgimiento de la malaria influida por la minería, primero en el Municipio de San Juan de Manapiare 47 y luego con mucha fuerza en el Municipio de Atabapo, que alcanzó una tasa de incidencia de 303 por mil, 250 veces más alta que la tasa nacional 38.

- Crecimiento poblacional en el Municipio Atures. El crecimiento periurbano desordenado y a lo largo de los ejes de carreteras convirtió al Municipio, donde se asienta la capital del Estado y la mayor parte de la población, en el primer productor de casos de malaria.

Descenso del PIB. Por último, jugó un rol muy relevante en el proceso descrito, un marcado descenso del PIB petrolero en el año 2003 (http://www.iadb.org/countries/indicators.cfm, accedido el 20/Sep/2006), como consecuencia de un paro petrolero en Diciembre del año 2002 que llevó al país casi a la quiebra, y que fue fuertemente influido por la polarización del país. Como puede apreciarse, la curva del PIB se inflexiona fuertemente en el 2003, y es cruzada por el número de casos de malaria que se duplican. Esto se asoció en Amazonas a carencia de recursos para movilizar al personal encargado del control de la malaria. Aunque el paro petrolero afectó a todo el país, su efecto en Amazonas en cuanto al flujo de recursos fue mucho más intenso y prolongado, lo cual se atribuye a que la contribución de Amazonas al PIB es casi nula 51. Nuevamente se observa que las poblaciones más deprimidas son más vulnerables, y que Amazonas terminó pagando un costo en enfermedad y muertes que el país nacional no conoció (Figura 3). 


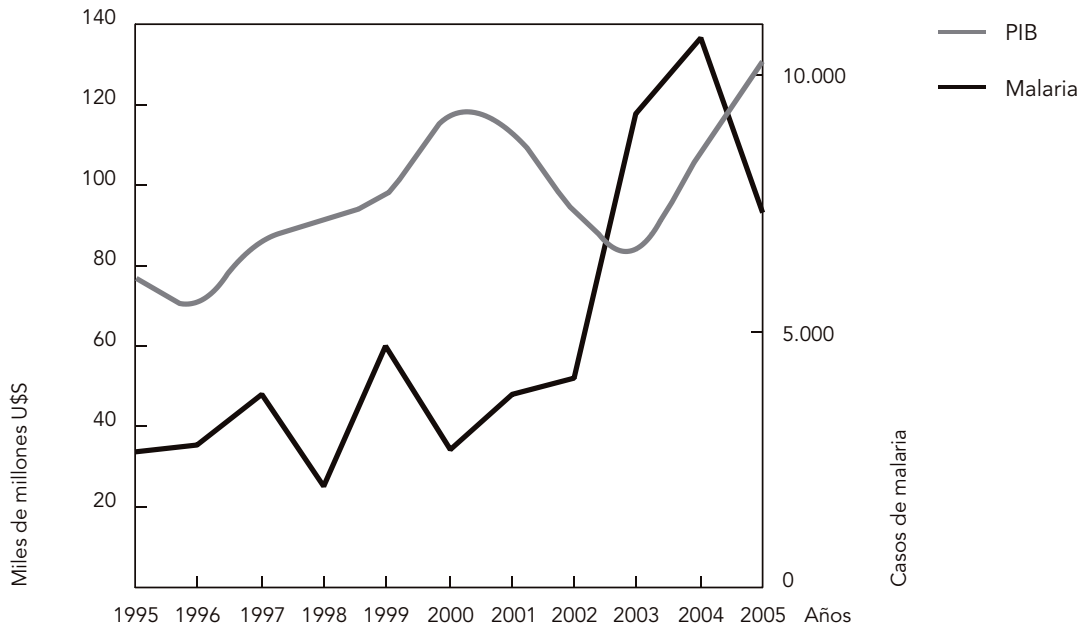

Fuente: Ministerio de la Salud y Desarrollo Social 40

\section{Conclusiones}

La brecha entre los países pobres y ricos se ha agudizado en la última década, y lo mismo ha sucedido con las asimetrías en la distribución de los recursos y los indicadores de salud entre diferentes regiones de un mismo país.

Las políticas públicas dirigidas hacia las Américas, que se expresaron en la década pasada en la reforma del estado y la liberalización del mercado, condujeron a un estancamiento o franco retroceso en los indicadores de desarrollo humano de la región. Se destaca que a la hora de impulsar estas reformas no se tuvieron en cuenta las limitaciones estructurales que impiden el crecimiento económico y el desarrollo humano 4.Un nuevo pacto mundial para el desarrollo sostenible de la aldea global es necesario. Un paso hacia él parece estar representado por la Declaración del Milenio, que reconoce que la pobreza, el hambre y las enfermedades tropicales no son un problema de las naciones pobres, sino de la toda humanidad y el compromiso de Monterrey que establece metas claras para países pobres y ricos. En este sentido, la globalización de los valores ayuda a establecer un balance frente a la globalización del capital, de las enfermedades y de las guerras preventivas.

La población indígena de Venezuela exhibe todavía en algunas áreas tasas de mortalidad infantil cercanas al promedio de los países más desfavorecidos del planeta. Las enormes diferen- cias en los indicadores de salud pública entre el Amazonas venezolano y el resto del país, esconde diferencias mayores aún dentro del estado, y aún dentro de una misma población indígena con distintos grados de acceso a los servicios de salud. Los programas de control de malaria, oncocercosis y otras enfermedades endémicas no han logrado sus metas, debido a una combinación de falta de recursos, excesivo centralismo y falta de adecuación a la realidad indígena.

La situación de desventaja de los pueblos indígenas en sus condiciones de salud con otros segmentos de la sociedad ha sido enfatizada y es recurrente en otras regiones de las Américas y la falta de adecuada data epidemiológica contribuye a la "invisibilidad" de este problema de salud 40 .

Como señala Stiglitz 48 el debate no gira en torno "globalizar o no globalizar", en tanto esta es una realidad que no se puede eludir. La constatación más fuerte es que los beneficios de la globalización son acumulados de manera desproporcionada por los más favorecidos y sus efectos negativos son sufridos más intensamente por las poblaciones más vulnerables 52 . Las preguntas relevantes en todo caso están vinculadas al análisis del impacto desigual de los cambios en los niveles locales y a saber si un modelo alternativo puede dirigir sus fuerzas para reducir la inequidad y el hambre y minimizar sus impactos sobre la salud pública. 


\section{Resumen}

La globalización económica ha acentuado la desigualdad y la vulnerabilidad a las enfermedades tropicales en todo el mundo. La población del Amazonas venezolano y particularmente la población indígena del interior del Estado es uno de los segmentos más desfavorecidos de las Américas a juzgar por los indicadores de salud. Las tasas de mortalidad infantil observadas en poblaciones indígenas en áreas remotas indican que han sido afectadas por la globalización de las enfermedades, pero no se han beneficiado de la globalización de la salud. Por otra parte, la globalización ha influido en las políticas públicas lo cual afectó la eficiencia de los programas de control de enfermedades tropicales en Amazonas. Un nuevo pacto mundial para el desarrollo sostenible de la aldea global es necesario, a través de la "globalización" de los valores y los derechos comunes de la humanidad. En Venezuela, nuevas politicas dirigidas hacia el subsector de salud indígena, más recursos y mayor autonomía de acción pueden ayudar a reducir las inequidades aquí descritas en el Amazonas venezolano.

Enfermedades Transmisibles; Ecosistema Amazónico; Políticas Públicas

\section{Referencias}

1. Secretaría Ejecutiva, Comisión Económica para América Latina y el Caribe. Globalización y desarrollo. Santiago de Chile: Comisión Económica para América Latina y el Caribe; 2002. (Documento LC/G2157 SES.29/3).

2. Programa de las Naciones Unidas para el Desarrollo. Informe sobre desarrollo humano en Venezuela. Caracas: Programa de las Naciones Unidas para el Desarrollo; 2002.

3. Feo-Isturiz O. Reflexiones sobre la globalización y su impacto sobre la salud de los trabajadores y el ambiente. Ciênc Saúde Coletiva 2003; 8:887-96.

4. Programa de las Naciones Unidas para el Desarrollo. Informe sobre desarrollo humano global. Caracas: Programa de las Naciones Unidas para el Desarrollo; 2003.

5. Follér M-L. Interactions between global proceses and local health problems. A human ecology approach to health among indigenous groups in the Amazon. Cad Saúde Pública 2001; 17 Suppl: 115-26.

6. Saker L, Lee K, Cannito B, Gilmore A, CampbellLendrum D. Globalization and infectious diseases. A review of linkages. Geneva: World Health Organization; 2004.

\section{Colaboradores}

C. Botto-Abella elaboró la versión inicial, y redactó la versión final del trabajo. B. Graterol-Mendoza contribuyó a la elaboración de la sección sobre globalización y políticas públicas en el Amazonas venezolano y a la edición del manuscrito final.

\section{Agradecimientos}

Al Laboratorio de Ciencias Sociales (LACSO/Venezuela) por su invitación a participar en el Taller sobre Globalización y Resurgimiento de las Enfermedades Tropicales (Angra do Reis, Brasil, 2003), al Fondo Nacional de Ciencia, Tecnología e Innovación (Proyecto ICM-Centro Amazónico de Investigación y Control de Enfermedades Tropicales "Simón Bolívar") y a Miguel Hernández (Dirección de Epidemiología y Análisis Estratégico) por el apoyo prestado, y a Magda Magris, José Antonio Kelly y Gregorio Sánchez por sus comentarios críticos.
7. Aublin L. Prefacio. In: Crespo LA, editor. Soñadores del Sur. Humanistas franceses en la selva venezolana. Biarritz: Atlántica Seguiré; 2000. p. 9-10.

8. León-Portilla M. Crónicas indígenas: la visión de los vencidos. Madrid: León Portilla; 1985.

9. Black FL. Infecção, mortalidade e populações indigenas: homogeneidade biológica como possível razão para tantas mortes. In: Santos RV, Coimbra Jr. CEA, organizadores. Saúde e povos índígenas. Rio de Janeiro: Editora Fiocruz; 1994. p. 63-90.

10. Anderson R, May R. Infectious diseases of humans. Oxford: Oxford University Press; 1991.

11. Yarzabal L, Layrisse M. El Estado de salud de la población yanomami. In: Arvelo-Jiménez N, editor. La reserva de biosfera yanomami: una auténtica estrategia para el desarrollo nacional. Caracas: Instituto Venezolano de Investigaciones Científicas; 1983. p. 80-116.

12. Mondolfi A. Consideraciones demográficas y epidemiológicas. In: Colchester M, Fuentes E, editores. Los Yanomami venezolanos: propuesta para la creación de una Reserva Indígena. Caracas: Edicanpa; 1983.

13. Botto C. La situación de salud de la población yanomami. Iglesia en Amazonas 1991; 54-5:11-5. 
14. Cook G. Manson's tropical diseases. 20th Ed. London: WB Saunders; 1996.

15. Cunill-Grau P. Geografía del poblamiento venezolano en el siglo XIX. 2a Ed. Caracas: Comisión Presidencial V Centenario, Facultad de Humanidades y Educación, Universidad Central de Venezuela; 1999.

16. Gadelha P. Conforming strategies of public health campaigns to disease specificity and national contexts: Rockefeller Foundation's early campaigns against hookworm and malaria in Brazil. Parassitologia 1998; 40:159-76.

17. Birn AE. Erradication, control or neither? Hookworm vs malaria strategies and Rockefeller Public Health in Mexico. Parassitologia 1998; 40:137-48.

18. Packard RM. "No other logical choise": global malaria eradication and the politics of international health in the post-war era. Parassitologia 1998; 40:217-30.

19. Gabaldón A, Berti AL. The first large area in the tropical zone to report malaria eradication: North Central Venezuela. Am J Trop Med Hyg 1954; 3: 793-807.

20. Almeida C. Reforma de sistemas de servicios de salud y equidad en América Latina y el Caribe: algunas lecciones de los años 80 y 90. Cad Saúde Pública 2002; 18:905-25.

21. Blas E, Hearst N. Health sector reform and equity - learning from evidence? Health Policy Plan 2002; 17 Suppl:1-4.

22. Brundtland GH. The World Health Report 2002: reducing risks, promoting healthy life. Geneva: World Health Organization; 2002.

23. Botto C, Graterol B, Hidalgo G, Gómez J, Perdomo A, Morales ME, et al. Environment, cultural change and public health in the venezuelan amazonas. http:/ / www.idrc.ca/forum3003/ev-26396-201-1DO_TOPIC (accedido el 29/Sep/2006).

24. Freire G. Piaroa enviroment and society in transition. A study of land use and social change in Middle Orinoco, Venezuela [PhD Thesis]. Oxford: University of Oxford; 2002.

25. Santana-Nazoa A. La cuestión científica y tecnológica en el Amazonas venezolano: evaluación y perspectivas. Caracas: Universidad Central de Venezuela; 1991.

26. Llambí L. Niveles de pobreza y manejo del ambiente en la Orinoquia venezolana. In: Escobar G, editor. Pobreza y deterioro ambiental en América Latina. México DF: Fondo Regional de Tecnología Agropecuaria; 2003. p. 127-51.

27. Briceño-León R. Bienestar, salud pública y cambio social. In: Briceño-León R, Minayo MCR, Coimbra Jr. CEA, editores. Salud y equidad: una mirada desde las ciencias sociales. Río de Janeiro: Editora Fiocruz; 2000. p. 15-24.

28. Esteves J, Dumith D. Diversidad biológica en Amazonas. Bases para una estrategia de gestión. Caracas: Fundación Polar/Programa de las Naciones Unidas para el Desarrollo/SADA-Amazonas; 1998.

29. Fajardo-Cortez V, Lacabana M. Ajuste estructural y mercado de trabajo, Venezuela 1989-1993. Cuadernos del CENDES 1993; (23):95-130.
30. Llambí L. Agricultura, ambiente y pobreza en la frontera noroccidental del Amazonas venezolano. Informe Final Laboratorio de Estudios Rurales y Agrícolas. Caracas: Instituto de Investigaciones Científicas; 2001.

31. Mascareño C. Balance de la descentralización en Venezuela: logros, limitaciones y perspectivas. Caracas: Instituto Latinoamericano de Investigaciones Sociales/Nueva Sociedad/Programa de las Naciones Unidas para el Desarrollo; 2000.

32. Arvelo-Jiménez N. La saga de los Yanomami - Reflexiones en torno al libro "Darkness in El Dorado". Interciencia 2001; 26:32-8.

33. Alvarado-Chacín N. Gestión social, pobreza y exclusión en Venezuela a la luz de las misiones sociales. Balance y perspectiva (2003-2004). Revista Venezolana de Análisis de Coyuntura 2004; 10:25-56.

34. España LP. Dos décadas de empobrecimiento y pobreza en Venezuela. Revista SIC 1997; (600): 480-3.

35. Oficina Central de Estadística e Informática. Informe sobre desarrollo humano: caminos para superar la pobreza. Caracas: Oficina Central de Estadística e Informática, Programa de las Naciones Unidas para el Desarrollo; 2001.

36. Ministerio de Salud y Desarrollo Social. Indicadores básicos de Salud 1999-2000. Caracas: Dirección General de Epidemiología y Análisis Estratégico, Dirección de Información Social y Estadísticas, Ministerio de Salud y Desarrollo Social; 2003.

37. Ministerio de Salud/Instituto Nacional de Estadística. Boletín estadístico de salud, Estado Amazona. N. 2. Puerto Ayacucho: Ministerio de Salud; 2006.

38. Ministerio de Salud y Desarrollo Social. Alerta epidemiológica. Programa de erradicación de la malaria. Caracas: Ministerio de Salud y Desarrollo Social; 2003.

39. Ministerio de Salud y Desarrollo Social. Informe de la dirección de epidemiología y análisis estratégico. Caracas: Ministerio de Salud y Desarrollo Social; 2003.

40. Santos RV, Coimbra Jr. CEA. Cenários e tendências da saúde e da epidemiologia dos povos indígenas no Brasil. In: Coimbra Jr. CEA, Santos RV, Escobar AL, organizadores. Epidemiologia e saúde dos povos indígenas no Brasil. Rio de Janeiro: Editora Fiocruz/ABRASCO; 2003. p. 13-47.

41. Kelly JA. Relations within the health system among the Yanomami in the upper Orinoco, Venezuela [PhD Thesis]. Cambridge: University of Cambridge; 2003.

42. Magris M, Rubio-Palis Y, Ruiz B, Frías D, Lines J. Insecticide treated nets for the control of malaria among the yanomami population of southern Venezuela: I. Epidemiological evaluation. In: XV International Congress for Tropical Medicine and Malaria. v. II. Cartagena: International Federation for Tropical Medicine; 2000. p. 113.

43. Centro Amazónico de Investigación y Control de Enfermedades Tropicales "Simón Bolívar". Informe anual. Puerto Ayacucho: Centro Amazónico de Investigación y Control de Enfermedades Tropicales “Simón Bolívar”; 1998. 
44. Rodríguez-Ochoa G. Situación de salud en el Territorio Federal Amazonas, Venezuela. In: Yarzabal L, Espinal C, Aragón L, editores. Enfoque integral de la salud humana en la Amazonía. Caracas: Universidad Central de Venezuela, Asociación de Universidades Amazónicas; 1992. p. 407-28. (Serie Cooperación Amazónica, 10).

45. Toro-Landaeta J. Problemática sanitaria de los pueblos indígenas del Amazonas. Iglesia en Amazonas 1993; 62-3:40-3.

46. Armada F. Aproximación a la situación sanitaria del Estado Amazonas. In: Chiappino J, Alès C, editores. Del microscopio a la maraca. Caracas: Ex Libris; 1997. p. 293-316.

47. Botto C, Duarte C, Perdomo A, Noya O, Pabón R, Lander O, et al. Influencia de la minería de oro en San Juan de Manapiare, estado Amazonas, sobre la transmisión de la malaria. In: De Lisio A, editor, II Conferencia Internacional Amazonía 21: logros para una agenda sustentable. VI Asamblea UNAMAZ. Caracas: Universidad Central de Venezuela/ Asociación de Universidades Amazónicas; 2000. p. 329. (Serie Cooperación Amazónica, 23).
48. Toro M. Economía y salud en el Amazonas venezolano. In: Chiappino J, Alès C, editores. Del microscopio a la maraca. Caracas: Ex Libris; 1997. p. 317-36.

49. Stiglitz JE. Pobreza, globalización y crecimiento: perspectivas en torno a algunos de los vínculos estadísticos. Caracas: Programa de las Naciones Unidas para el Desarrollo; 2003.

50. Stiglitz JE. El malestar en la globalización. Madrid: Taurus; 2002.

Recibido el 11/Nov/2006

Versión final presentada el 06 /Oct/2006

Aprobado el 23/Oct/2006 